\title{
Soberanía territorial, conservación ambiental y comunidades de campo común en Chile central
}

\author{
Jorge Razeto Migliaro \\ Universidad de Chile, Santiago, Chile. \\ Email: jrazeto@uchile.cl \\ Emilia Catalán Martina \\ Investigadora independiente, Santiago Chile. \\ Email: emiliacatalan@gmail.com \\ Juan Carlos Skewes Vodanovic \\ Universidad Alberto Hurtado, Santiago, Chile. \\ Email: jskewes@uahurtado.cl
}

\begin{abstract}
Resumen:En las serranías de las cordilleras de los Andes y de la Costa de Chile central, se emplazan comunidades de campo, poseedoras de propiedad colectiva. El presente trabajo analiza el rol de la propiedad comunitaria como garante de la conservación de los ecosistemas mediterráneos, a la luz de la teoría de los comunes, la ecología política latinoamericana y las propuestas del giro ontológico.Se analiza la realidad de tres comunidades de campo de la región de Valparaíso, tomando como base estudios etnográficos. Frente a las amenazas que aquejan a las comunidades -minería, monocultivos, urbanización, - el campo común como territorio indiviso e intransferible constituyente el soporte vinculante de la relación entre comuneros y su entorno, expresado en sus prácticas productivas -principalmente ganadería- como en novedosas prácticas de conservación comunitaria, los cuales permiten el ejercicio de la soberanía sobre el territorio, en tanto forma de resistencia ambiental, social y cultural.
\end{abstract}

Palabras claves: Comunidades agrícolas; propiedad colectiva;conservación comunitaria;soberanía territorial

\section{Territorial sovereignty, environmental conservation and common-field communities in central Chile}

\begin{abstract}
In the mountain chains of the Andes and the Coast in Central Chile, are located land communities, owners of collective property. This paper analyzes the role of community property as guarantor of the conservation of Mediterranean ecosystems, in light of the theory of the commons, Latin American political ecology and the proposals of the ontological turn. The reality of three field communities in the Valparaíso region is analyzed, based on ethnographic studies.

Facing the threats that affect the communities - mining, monocultures, urbanization, - the common land as undivided and non-transferable territory constitutes the binding support of the relationship between communal farmers and their environment, expressed in their productive practices -principally livestock- as in novel practices of community conservation, which allow the exercise of sovereignty over the territory, as a form of environmental, social and cultural resistance.
\end{abstract}

Keywords: Agricultural communities; common property; community conservation; territorial sovereignty. 


\section{Soberania territorial, conservação ambiental e comunidades com terra comum no centro do Chile}

Resumo: Nas cordilheiras dos Andes e na costa central do Chile vivem as poucas comunidades rurais proprietárias de propriedade coletiva. Este artigo analisa o papel da propriedade da comunidade como garantia de conservação dos ecossistemas mediterrâneos, à luz da teoria dos commons, a ecologia política latino-americana e pelas propostas do giro ontológico.Uma abordagem etnográfica de três comunidades na região de Valparaíso destaca a importância dos bens comuns para a preservação da biodiversidade.

Diante das ameaças que afligem as comunidades - mineração, monoculturas, urbanização - o bem comum, como propriedade intransferível e indivisível, constitui o suporte vinculante da relação entre habitantes da comunidade e seu entorno, expressado nas suas práticas produtivas - principalmente criação de gado- como em inovadoras práticas de proteção ambiental, os quais permitem o exercício da sua soberania territorial, enquanto exercem resistência ambiental, social e cultural.

Palavras-chave: Comunidades agrícolas; propriedade comum; conservação comunitária; soberania territorial.

\section{Introducción}

En su texto clásico, Hardin invita a imaginar un pastizal al que cada pastor trata de llevar la mayor cantidad de animales que puede hasta desencadenar el único desenlace posible: la tragedia de los comunes. La imagen que ofrecenla comunidad de campo de la Serranía del Ciprés, las comunidades aledañas al Parque Nacional de La Campana y la comunidad de campo de Jahuel en Chile central cuestionan el sentido de lo planteado por Hardin. Mientras que en los valles la privatización de los campos no solo eliminó a los comunes, sino que también los resabios del bosque mediterráneo, en los cerros colindantes, donde subsisten las comunidades de campo, reverdecen los comunes y, a pesar de ciertos escepticismos ecológicos, con vacas incluidas. De aquí quela pregunta que inspira este artículo problematiza en torno a las formas como la gestión colectiva de los recursos, amparada en la figura de las comunidades de campo, ha hecho posible la protección del bosque nativo en territorios profundamente intervenidos por la expansión de una economía extractiva fundada en la privatización de los recursos, la que, por el contrario, lo ha eliminado.

Entre las múltiples claves para comprender el papel que las comunidades de campo juegan en la protección del bosque nativo, los aportes de Elinor Ostrom resultan especialmente útiles. El acceso a los recursos nunca es lo abierto que Hardin supone. Más aún, las perspectivas teóricas actuales en antropología apuntan a entenderlos como simultáneamente constituidos y constituyentes de los procesos socioambientales. El entramado social se articula en la relación entre seres humanos y otros-que-humanos. Ostrom incorpora la dimensión institucional para dar cuenta de los impactos ambientales de las actividades humanas. De este modo abre las puertas para buscar herramientas de cooperación en el enfrentamiento de los desafíos ecológicos del tiempo contemporáneo. Una de las contribuciones de Ostrom a la economía ha sido, justamente, superar la dicotomía público-privada en la identificación de los bienes comunes y, con ello, reconocer que existen formas colectivas de uso y explotación decampos de pastoreo que resuelven el dilema planteado por la tragedia de los comunes. Su planteamiento involucra, desde el punto de los actores, la negociación de costos y beneficios en la regulación al acceso a los recursos. Ni privatización ni regulación aparecen en esta mirada como las dimensiones necesarias para resolver la gestión común, sino que, por el contrario, lo que resulta especialmente gravitante es el piso institucional que compromete a cada una de las partes involucradas en la gestión del bien común. Este enfoque de trabajo ha sido fundamental en el estudio de la conservación de los ecosistemas forestales, siendo un campo privilegiado para el estudio de los bienes comunes, dada la extensión mundial de formas comunitarias de gestión de bosques, particularmente en el llamado Tercer Mundo, donde existe mayor porcentaje de propiedad comunitaria, lo cual da cuenta de la importancia de estos ambientes para la subsistencia de poblaciones rurales a nivel mundial (Agrawal, 2007).

Si bien el paradigma de Ostrom se inscribe en el marco de una visión moderna de la relación con la naturaleza, no resulta conveniente desprenderse del todo de sus planteamientos sino que más bien revisitarlos a 
la luzdel amplio campo de laecología política, particularmente en sus cruces con las perspectivas antropológicas que plantean el giro ontológico y el giro decolonial Alimonda, 2016; Alimonda, Toro Pérez y Martín, 2017; Escobar, 2016; Heywood et al., 2017; . Ello resulta especialmente necesario cuando se asume la posibilidad de que los arreglos colectivos socioambientales estén sujetos a otras definiciones de la realidad y, más aún, a otras formas de constituir lo real(Descola, 2013). El posicionamiento de los comuneros en su medio no necesariamente fluye de cálculos utilitaristas sino de formas de asociatividad con los animales, las montañas, el agua y las especies vegetales. Una forma de entender su constitución como sujetos colectivos arranca de su vínculo más sustantivo en el paisaje del que son parte y que contribuyen a recrear. Así, las relaciones comunitarias se expresan en la propiedad colectiva y van más allá de las relaciones solo entre humanos. Bajo esta lectura, la expresión "gestión de bienes comunes" es inexacta para las relaciones recíprocas que se generan entre los seres de un territorio. Las acciones que se suceden en estos espacios también trascienden la división de los ámbitos político, económico y cultural, segmentación propia de los sistemas modernos liberales (Patzi, 2004). Esta forma de organización local del territorio ilustra de buena manera la ilusoria de la dicotomía naturaleza-cultura, en aspectos tales como la configuración del paisaje de la comunidad, las actividades productivas que allí se realizan y las motivaciones de la defensa de su territorio. El paisaje es fruto de una simbiosis informada tanto por posicionamientos recíprocos entre los seres vivos como por las relaciones de poder entre los propios seres humanos (Escobar, 2010).

En América Latina, son significativos los estudios de comunidades bajo los lentes de la ecología política (Escobar, 2010, 2016; Leff, 2017) como lo son aquellos que se refierena la comunalidad (Bautista Segales, 2014; Patzi, 2004; Rivera Cusicanqui, 2017). En Chile, no obstante, el tema ha estado más restringido a las zonas norte y sur del país donde la territorialidad indígena ha concitado la mayor atención. En Chile central, la mayoría de los estudios se han enfocado en las comunidades de la región de Coquimbo, en la que se concentran las comunidades agrícolas (Castro y Bahamondes, 1986; Livenais y Aranda, 2003), destacando aquellos enfocados en la identidad propia de estas realidades (Erazo y Garay-Flühmann, 2011; Gajardo, 2014). En el caso de las comunidades de la región de Valparaíso existen escasas publicaciones, prevaleciendo las revisiones históricas (Baraona, Aranda y Santana, 1961; Góngora y Borde, 1956; Venegas, 2009), y recientes lecturas que profundizan la lógica relacional que se expresa en las comunidades con propiedad colectiva (Catalán, 2015; Razeto, 2016).Las comunidades de campo en Chile central, asociadas usualmente a territorios montañosos, poseen diversos orígenes y formas de tenencia, así como distintas características socioculturales y ambientales. Todas ellas, no obstante, establecen una particular dependencia respecto de los recursos de su campo común.

El presente artículo secentrará en el caso de comunidades de campo correspondientes a las serranías de las cordilleras de los Andes y de la Costa de la región de Valparaíso. Nos enfocaremos en el rol particular que estas comunidades poseen en la conservación del ecosistema mediterráneo, caracterizado en Chile por la presencia de matorral y bosque esclerófilo. A nivel mundial, estos tipos de ecosistemas representan menos del 5\% de la superficie terrestre (Cowling, Rundel, Lamont, Arroyo y Arianoutsou, 1996).En el caso de Chile se han visto fuertemente impactados por la influencia antrópica (Schulz, Cayuela, Echeverría, Salas y Rey Banayas, 2010), vinculada al monocultivo frutícola y forestal, la minería, la urbanización, entre otras, lo que se suma a la escasa protección formal en términos de áreas protegidas (Simonetti, 1999; Tognelli, Ramírez de Arellano y Marquet, 2008).

En este escenario es sugerente elpapel que las comunidades de campo desempeñan en la protección del bosque mediterráneo. Se trata de comunidades campesinas forjadas en torno a la tenencia en común de campos de pastoreo, tenencia que les fue reconocida por el Estado como propiedad colectiva en 1967 (Solís de Ovando, 2004). En la actualidad muchas de estas comunidades no solo han luchado para mantener las tierras de propiedad común, sino que además se han esmerado por aportar a la conservación del matorral, del bosque esclerófilo y otros ecosistemas asociados, que se encuentran en estado crítico por la acción antrópica derivados de un modelo de desarrollo que privilegia la propiedad privada. La experiencia de las comunidades da cuenta de formas de protección que no necesariamente se condicen con losmodos clásicos de conservación fundados en la exclusión de la actividad humana (Cox y Underwood, 2011). A objeto de analizar estas formas de protección y el papel que la gestión colectiva del bien común juega en su implementación se revisa el caso de tres comunidades de campo de la Región de Valparaíso: en la Cordillera de la Costa, la comunidad de Las Palmas del Niño 
Dios, aledaña al Parque Nacional La Campana en la comuna Olmué, y, en la Cordillera de los Andes, las comunidades de campo de El Asiento y Jahuel, ubicadas en las comunas de San Felipe y Santa María respectivamente. Se analizan los contextos actuales que las revitalizan, particularmente los factores que las han llevado a proteger su territorio, renovando y potenciando su identidad, y en distinta medida, su autonomía, ejercida a partir del argumento de la conservación.

A continuación, se presenta, en primer lugar, los materiales de la investigación, incluyendo la descripción de las comunidades de campo y la metodología a través de la que se abordaron los casos estudiados. El apartado de resultados que sigue se concentra en las tres comunidades que constituyen la muestra del estudio, cuya comparación se desarrolla en la discusión. Las conclusiones de este artículo apuntan, por una parte, a reconsiderar la relación entre la propiedad individual y la colectiva en la perspectiva de la protección de los bienes comunes y, por la otra, a valorar la contribución que las comunidades de tierra hacen a la conservación de la rica biodiversidad del bosque mediterráneo.

\section{Materiales y métodos}

\section{El universo: Las comunidades de campo}

Las comunidades de campo (formalmente llamadas Comunidades Agrícolas), son una forma colectiva particular de tenencia cuyo origen, según Vergara (2005), es posible rastrear desde los tiempos de la colonia en el siglo XVI. La versión oficial del Ministerio de Bienes Nacionales, establece que:

Las Comunidades Agrícolas tienen su origen en el otorgamiento de mercedes de tierra a los conquistadores durante la Colonia, las cuales fueron pasando de generación en generación a sus habitantes, manteniéndose unidas a través de costumbres y tradiciones vinculadas al medio ambiente en el cual se desarrollaron (Ministerio de Bienes Nacionales, s.f.).

La corona española, a través de la figura de autoridad de gobernador general, concedió una relativa cantidad de mercedes de tierras a oficiales y personal de tropa, en retribución a los servicios prestados al Reino especialmente en los albores del siglo XVI; no obstante, en el norte verde de Chile y en las zonas montañosas andinas del centro, tierra de muy baja productividad por su condición semiárida y agreste, fue asignada a militares de bajo rango o emprendedores empobrecidos, que sin poseer grandes recursos ni contactos, no conformaron procesos sustantivos de control sobre las mencionadas asignaciones de tierra, siendo muchas de ellas abandonadas tempranamente por sus asignatarios originales (Razeto, 2016). Por consecuencia, se constituyeron en refugios para mineros y ganaderos pobres que sin soporte legal, ocuparon vastas extensiones de tierra por centenas de años, constituyendo inicialmente "comunidades de hecho" sin reconocimiento formal (Razeto, 1986): “Mestizos sin mayor vinculación étnica formal, solo pobres 'naturalizados' y asimilados, peones errantes, ocupantes de facto de terrenos aparentemente de nadie, cuya figura genérica se mantiene relativamente estable durante varios siglos” (Razeto, 2016, p.56). Ello acompaña de manera paralela y marginal, el control de las tierras privilegiadas que conformaron la soberanía aristocrática y monolítica en torno a las grandes haciendas y latifundios durante más de 300 años. Algunas comunidades son también resultado de los conflictos de deslindes con la gran propiedad, la que progresivamente se fue apropiando de las mejores tierras de riego, "expulsando a las comunidades hacia las rinconadas y serranías; tierras de mala calidad, con pendientes excesivas y con agudos problemas de erosión agravado por el sobretalajeo de ganado menor y la destrucción del matorral para la consecución de combustible” (Gatica y Thayer, 1970, p. 119). De esta manera, asumimos que no existe una sola explicación de origen para estas comunidades, sino que responden a situaciones particulares y diversas; a veces por abandono, a veces por ocupaciones centenarias que devinieron en propiedad, a veces por sesión de antiguos grandes propietarios, o por conflictos entre vecinos o herederos, que devinieron en despojo.

No es sino avanzado el siglo XX, que esta realidad fue reconocida y asumida por el Estado, en tanto su administración política resuelve otorgarle reconocimiento legal a una parte de ellas, por medio de una política pública que se implementa progresivamente, que culmina y se consolida en el Decreto con Fuerza de Ley $\mathrm{N}^{\circ}$ 5, 
dictado el 26 de diciembre de 1967, modificado por la Ley № 19.233 en 1993, cuya vigencia se proyecta hasta la actualidad. De esta forma se asigna tierra y se reconoce la propiedad efectiva de los habitantes que ocupaban un determinado territorio, constituyendo comunidades agrícolas en las regiones de Coquimbo, Valparaíso y Metropolitana, hasta que expiró el plazo de vigencia para acogerse a sus beneficios el 5 de agosto de 1998 (Solís de Ovando, 2004).

El procedimiento incluyó la incorporación en calidad de propietarios a las familias residentes de manera permanente, con ascendencia de al menos dos generaciones efectivas en el lugar, ejecutoras de actividades agrícolas y/o ganaderas en pequeña escala. De esta manera se establecía un grupo de comuneros y un territorio concreto asociado, que normalmente adoptaba el nombre tradicional del lugar. Esta figura solo permitía la transferencia de un derecho inicial hacia los herederos reconocidos legalmente, una vez fallecido el titular asignatario de derechos iniciales, razón que facilitó la nominación alternativa de "comunidades sucesoriales" (Razeto, 2016).

Si bien la ley se formaliza en la década del 60, no es sino a partir del retorno de la democracia en 1990, que la normativa establece en su artículo $4^{\circ}$, que el Ministerio de Bienes Nacionales debía asesorar jurídicamente en su fase de constitución, en forma gratuita a todas las comunidades que hubieran constituido e inscrito un predio común a su favor, respecto a su dominio o explotación y derechos de aprovechamiento de aguas, logrando con ello la consolidación de la figura de propiedad colectiva que había sido cuestionada, así como una actualización o saneamiento de los títulos de propiedad que a esa fecha al menos existían (Razeto, 2016). No obstante, es necesario mencionar que no todas las comunidades históricas realizaron este proceso de regularización, manteniéndose algunas informales, u otras, acudiendo a otras figuras jurídicas alternativas a la comunidad agrícola. Esta forma comunitaria se concentra preferentemente en la región de Coquimbo, en el llamado norte verde, zona semidesértica donde se reconoce la existencia actual de 187 comunidades, siendo bastante menor la concentración en las regiones de Atacama (2 comunidades), Valparaíso (7 comunidades) y Metropolitana (1 comunidad) (Ministerio de Bienes Nacionales, s.f.).

Así, algunas de estas propiedades en las inmediaciones de la cordillera de Los Andes y de la cordillera de La Costa en la Región de Valparaíso, en la cuenca superior e inferior del río Aconcagua. Estas se diferencian sustantivamente de las comunidades de la región Coquimbo, desde el punto de vista de las prácticas productivas, pero, sobre todo, por los contextos geográficos que ocupan, en la medida que solo existe este tipo de propiedades colectivas, en zonas de media y alta montaña, en lugares marginales a la historia hacendal de la región (Razeto, 2016; Venegas, 2009). Las mercedes de tierra asignadas durante la colonia en las zonas bajas y valles, tenían aptitud de riego, eran de alta productividad y constituyeron estancias ganaderas y luego haciendas muy prósperas, que conformaron la aristocracia chilena por excelencia (Bengoa, 1990). El resto quedó abandonado a los pobres, hasta ahora que se descubrieron "nuevas riquezas” en su interior. La diferenciación entre las tierras ricas y las tierras pobres, vista a la luz de esa época, puede entenderse a partir de Figura 1, donde las zonas irrigadas, correspondientes a cuencas de ríos y valles conformantes, corresponden a lugares de instalación y control de haciendas preferentemente de vocación agrícola, mientras las zonas montañosas corresponden a territorios agrestes mal controlados o desechados por las propias haciendas, donde solo se verifica aptitud ganadera estacional de carácter estival y comunitaria (Bengoa, 1990; Razeto, 2016). 
Figura 1

Planta y descripción visual del valle de Aconcagua. 1740, anónimo.

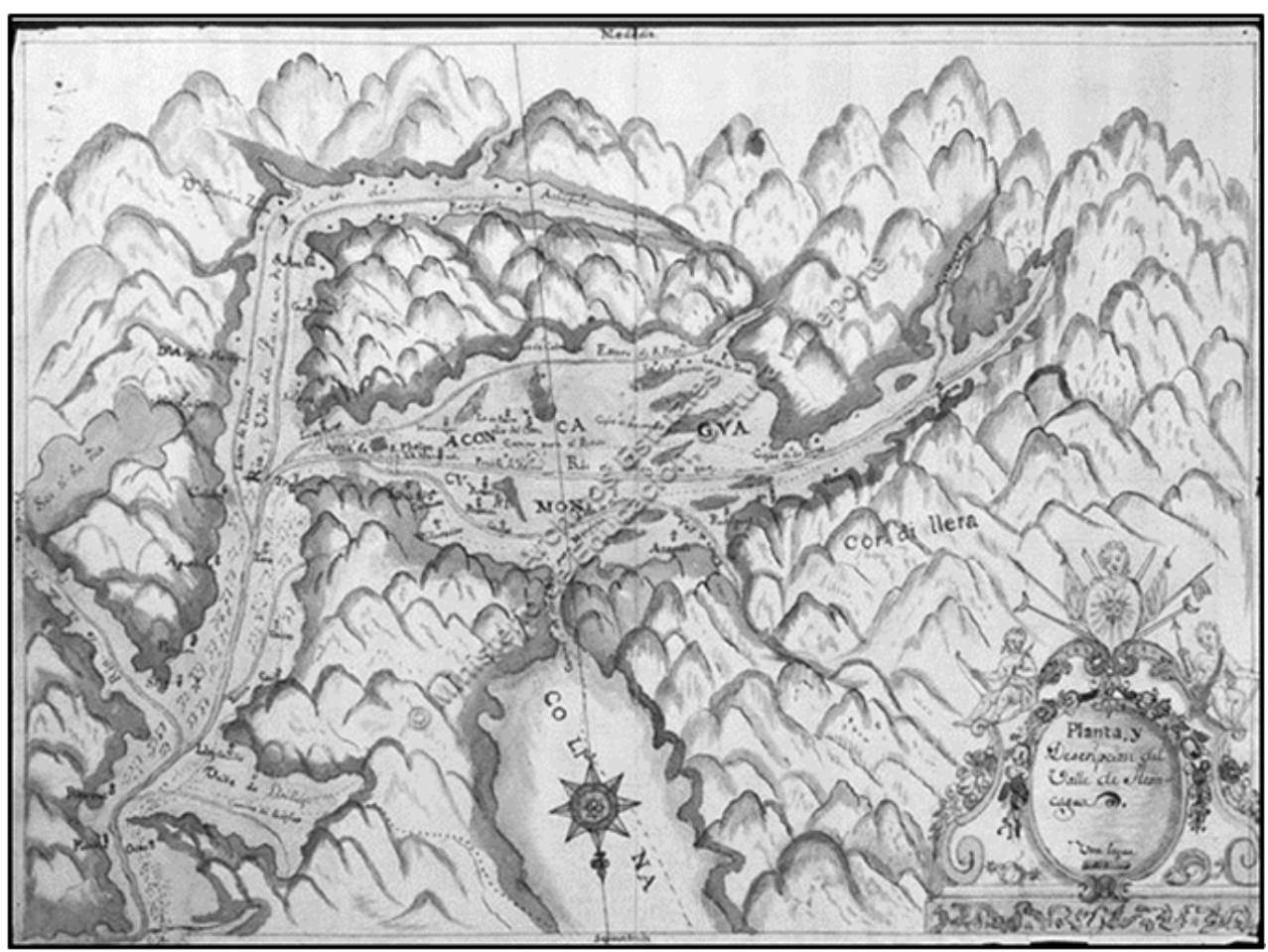

Fuente: Archivo General de Indias

Asociado a estas formas de propiedad colectiva, el origen de los sistemas ganaderos en esta zona montañosa se sitúa en la época colonial, con la adopción de un modelo de tradición europea enfocado principalmente al comercio de exportación, lo que es acompañado también por el incentivo al nacimiento de pequeños propietarios de animales, que posteriormente son reconocidos como ganaderos(Bengoa, 1990; Guerra, 2005; Razeto, 2016).

\section{Estudio de casos comparados}

El estudio de casos comparados que aquí se ha escogido como estrategia metodológica para dar cuenta de la experiencia de conservación de tres comunidades de tierra es consistente con las características de la relación entre el equipo de investigación y los territorios considerados (Stake, 2006). En efecto, esta aproximación permite un desarrollo de largo aliento, pudiendo establecer una comparación entre los casos considerados en un determinado contexto, que, en este caso, está representado por la expansión de las explotaciones mineras y agrícolas, y de desarrollo inmobiliario, en zonas de alto valor ecológico. Los casos de estudio presentados son, pues, producto de diversos procesos investigativos, poseedores de dimensiones académicas y aplicadas, en el marco de los cuales se han establecido relaciones de apoyo mutuo entre los investigadores y las comunidades. Si bien el trabajo en estos territorios no se ha limitado a un solo proyecto de investigación, estas tres comunidades fueron parte del proyecto FONDECYT “Antropología del Bosque: horizontes para una protección socialmente inclusiva de los bosques esclerófilos y templados de Chile”, desarrollado entre el año 2014 y el 2017 , entre cuyos objetivos se encontraba la identificación de prácticas consuetudinarias y su rol ecológico en el contexto de territorios con presencia de bosque nativo, particularmente de aquellas prácticas estratégicas para la convivencia entre seres humanos y no humanos. Justamente, las comunidades de campo se identificaron como un aporte a la convivencialidad entre humanos y no humanos.

Los procesos investigativos se basaron en etnografías en las tres localidades, lo cual incluye recorridos comentados por los espacios montañosos, entrevistas en profundidad y entrevistas grupales a cerca 
de 50 a comuneros y comuneras, además de grupos de discusión centrados en los avances de la investigación.

El análisis que se presenta a continuación se centra en la relación que cada una de las comunidades consideradas mantiene con su entorno, focalizándose la observación en las iniciativas de conservación que han surgido desde las propias comunidades. El trabajo por ellas desarrollado se articula con su vocación productiva por la ganadería trashumantey plantea iniciativas de conservación en un contexto de matorral y bosque esclerófilo, las que se han activado a partir de diversas coyunturas y que se han manifestado de distinta manera, como se verá a continuación.

\section{Resultados}

Actualmente existen siete comunidades agrícolas en la región de Valparaíso, y otras tantas no formalizadas. En los intersticios montañosos andinos de Chile central, es posible reconocer la existencia de al menos diez contextos comunitarios, los cuales se sitúan en vastas zonas con escasa población, en tierras altas y montañosas, áridas y ocupadas estacionalmente con fines ganaderos de escala reducida durante la época estival. Las zonas bajas albergan localidades pobladas de manera permanente, villorrios y pequeños pueblos donde parte de sus habitantes son comuneros, de la cual una parte aún menor realizan prácticas ganaderas, estimándose en una población actual de no más de 50 familias que se dedican a estas actividades trashumantes, mostrando una tendencia evidente a la reducción de la actividad respecto de décadas anteriores (Razeto, 2016). Es indicativo registrar que en la misma medida que se reduce la actividad ganadera trashumante, se expande la actividad industrial en formato de enclaves, especialmente mineros, hidráulicos y turísticos, que modifican la estructura de uso y la propiedad, trastocando los ejes de la actividad económica y progresivamente van invadiendo y amenazando los territorios comunitarios.

En la cordillera de la Costa se reconocen cinco comunidades de campo ubicadas en la comuna de Olmué (Venegas, 2009), a las que se suman otras comunidades históricas vecinas de la región Metropolitana (Brucher, 1962). Éstas se emplazan en las laderas orientales y occidentales, las cuales, a diferencia de la cordillera de Los Andes, se caracterizan por la abundante presencia de matorral y bosque esclerófilo. En las zonas más planas y/ o bajas se emplazan las localidades pobladas, mientras que el campo común se sitúa en las zonas altas, las cuales coinciden en buena medida con los espacios de ganadería transhumante local, que al igual que en el contexto andino, ha tendido a la baja (J.L. Díaz y E. Catalán, informe Diciembre del 2018, comunicación personal), mientras el territorio no comunitario ha tendido a su parcelación por la presencia de segundas viviendas (Hidalgo, Borsdorf y Plaza, 2009), con la tensión latente de instalación de grandes proyectos de infraestructura y minería.

En lo que sigue, revisaremos la realidad de tres casos de comunidades en diferentes contextos ecológicos y las formas de gobernanza que se han dado para gestionar su campo común frente a las amenazas que les plantea el contexto. No obstante, lo que poseen en común, es la defensa de la soberanía de su territorio y su comunidad, tomando como central en esta defensa la conservación de su entorno. Las ubicaciones relativas de cada comunidad, e iniciativas de conservación asociadas, pueden visualizarse en la Figura 2.

\section{Comunidad de Campo de Jahuel}

La Comunidad de Campo de Jahuel es un caso ejemplar de defensa del territorio por medio de la conservación. Ubicada en la parte alta (nor-oriente) de la comuna de Santa María, comprende alrededor de 8.200 hectáreas de serranías y cordilleras bajas de los Andes, cuyas alturas fluctúan entre los 1.500 y los $3.000 \mathrm{msnm}$. Se trata de tierras de propiedad comunitaria compuesta por 126 comuneros, cuyas familias se ubican preferentemente en un asentamiento semi-continuo, en las localidades de El Zaino, Jahuelito, Santa Filomena, cuya población total se acerca a los 2.000 habitantes. Si bien su origen comunitario se remonta a épocas muy antiguas, ha sufrido diversas afectaciones y enmiendas legales, siendo actualizada su vigencia en los años 90' del siglo pasado. 
Las actividades económicas locales principales son el cultivo de olivos y procesamiento de aceitunas, producción de tunas y otros frutales en menor escala en la parte baja de la zona. Una parte de ellos, preferentemente comuneros, realiza actividades ganaderas de carácter trashumante asociadas a las montañas, a través del manejo de ganado mayor (vacunos, mulares y caballares), con decreciente presencia caprina.

La conformación geográfica interna está conformada por 2 pequeñas cuencas hidrográficas correspondiente a bosque esclerófilo, de intensa actividad histórica y de gran biodiversidad. Estas sub-cuencas son el estero La Laja y el estero El Zaino, de una gran pendiente diferencial altitudinal que dan origen al Estero Jahuel. En la parte superior de la propiedad se encuentra la laguna El Copín, renombrado y mítico cuerpo de agua que alimenta y complementa un complejo y frágil ecosistema (Ciem Aconcagua, 2015a).

La historia local recuerda la convivencia con actividades mineras y pirquineras de bajo impacto, que en la actualidad se activa con el resurgimiento de dos concesiones de mediana escala: las mineras Paula y Aguas Claras, que establecen en las inmediaciones comunitarias, prácticas de sondeo y extracción, instalando faenas industriales y generando conflictos con la comunidad al utilizar y contaminar sus aguas. En este contexto, la comunidad de Jahuel y sus poblados han desplegado un interesante y diverso repertorio de acciones colectivas que incluyenla conservación como parte de su estrategia de defensa (Carvajal, 2014). La creación del Santuario de Naturaleza Zaino-Copín, que grava ambientalmente 6.700 hectáreas, es decir, casi el $80 \%$ de su propiedad colectiva, constituye su principal contribución a la protección del bosque esclerófilo, a la vez que consolida su vocación por la gestión colectiva de su patrimonio territorial.

\section{Comunidad Agrícola Serranía El Asiento}

Responde a una superficie relativamente reducida, cercana a las 2.000 hectáreas, en los entreveros del Cerro Tabaco, reconocido cordón montañoso de las comunas de San Felipe y Putaendo, que fluctúa entre los 1.200 y los $2.400 \mathrm{msnm}$, en las cercanías del poblado de El Asiento, cuyo distrito censal contabiliza cerca de 2.400 habitantes. La comunidad agrícola está compuesta por 106 comuneros de carácter muy diverso, ya que durante un proceso divisorio interno (en torno a los años 2000), se estableció un procedimiento de venta de derechos de tierra (haciendo uso de una facultad legal), lo que descompone la visión unitaria de sus comuneros propietarios, quedando solo una parte de las familias haciendo usufructo productivo de dichas tierras. El resto de los propietarios corresponde a personas que en general tienen residencia en la zona, pero no tienen un arraigo significativo. Ello lleva a reconocer una diversidad tan etérea como sociocultural, lo que conlleva algunas complejidades como también algunas potencialidades (Ciem Aconcagua, 2015b).

Las riquezas naturales del entorno se caracterizan por una compleja y vasta biodiversidad de flora y fauna, en tanto expresión de una vegetación esclerófila de fondo de quebrada boscosa, con laderas de exposición norte y sur muy diversas. El máximo atributo ambiental del predio, radica en un relicto de Ciprés de la Cordillera (Austrocedrus chilensis) compuesto por 450 ejemplares cuya longevidad ha sido definida en torno a los 1.700 años vivos; siendo los ejemplares más septentrionales y de mayor altitud del planeta (Ciem Aconcagua, 2015b).

En las inmediaciones de la quebrada, limitando con la propiedad colectiva, destaca la presencia de la minera Clarita, mediana industria cuya mantención y pretensión de expansión, amenaza directamente la calidad de vida del poblado de El Asiento y particularmente la riqueza ambiental de la quebrada, tanto por la extracción de sus aguas como por la emanación de material particulado de origen minero. Frente a ello, la comunidad ha emprendido diversas estrategias de defensa de su entorno, destacando el logro de la declaración del Santuario de Naturaleza Serranía del Ciprés el año 2006, correspondiente a más de 1.000 hectáreas de su propiedad. Como expresión de una compleja y frágil realidad socio-ambiental, la quebrada sufrió un gran incendio, que arrasó prácticamente la totalidad del Santuario, salvándose solo parte del relicto referido.

\section{Comunidad Las Palmas del Niño Dios}

La comunidad de Las Palmas del Niño Dios se ubica en el cordón montañoso de los cerros La Campana y el Roble, a unos 780 msnm, vecina del Parque Nacional La Campana. Su vegetación corresponde principal- 
mente a matorral y bosque esclerófilo, destacando la presencia de roble blanco (Nothofagus macrocarpa) y palma chilena (Jubaea chilensis). Estos elementos le dan gran importancia ecológica a su campo común. La comunidad es reconocida por la fiesta del Niño Dios de Las Palmas, a la cual peregrinan personas de múltiples comunidades aledañas, recorriendo los cerros, lo que representa la relación tradicional entre las comunidades serranas (Elórtegui y Moreira, 2009).

El campo común corresponde a 1900 hectáreas. En su constitución a mediados del siglo XX se inscribieron 102 comuneros. En 1998 formalizaron su situación, mediante la figura de una Corporación de Desarrollo de Derecho Privado y no como Comunidad Agrícola, en la que se inscribieron 200 comuneros. Actualmente habitan cerca de 396 personas, no obstante, un porcentaje importante de las viviendas -más de la mitad según los palminos- corresponde a parcelas de agrado o segundas viviendas (Catalán, 2015). Entre las principales actividades productivas, persisten algunas actividades tradicionales dependientes del campo común, como la ganadería, la extracción de tierra de hojas y la elaboración de carbón. La primera no posee importancia económica, pero sí cultural, y es realizada tanto por jóvenes como por personas mayores, calculándose la existencia de cerca de 400 cabezas de ganado en la comunidad (Díaz y Catalán, 2019, comunicación personal).

La principal presión sobre el territorio son las parcelas de agrado, principalmente por su alta demanda de agua -pozos profundos y extracción ilegal - y la subdivisión de las propiedades individuales en pequeñas parcelas, que facilita la tala del bosque nativo. La presencia de las parcelas de agrado es un fenómeno contradictorio para la comunidad. Por un lado, se estima la presencia de los afuerinos, tanto por su aporte económico -por la venta de terrenos individuales subdivididos de los comuneros y luego, por el empleo ofrecido como cuidadores de las parcelas de agrado-, como por su valorización de la naturaleza presente en el lugar. Por otro lado, pesan las consecuencias ambientales negativas, pero también las sociales, ya que los parceleros no comulgan con algunas de las costumbres de los comuneros -tales como la ganadería, mostrando molestia por la constante libre circulación de los animales. Para las y los palminos, la llegada de los parceleros da cuenta de su valioso entorno, el cual están decididos a conservar (influenciados también por Parque La Campana), pero a su modo: con sus costumbres, sin figuras de conservación formal, pero sí medidas suscritas a la institucionalidad de la comunidad, imponiendo su soberanía en el territorio, lo que ha significado regular la presencia de los parceleros, tensionando la relación individuos-colectivo.

Figura 2

Mapa Comunidades de campo común e iniciativas de conservación asociadas

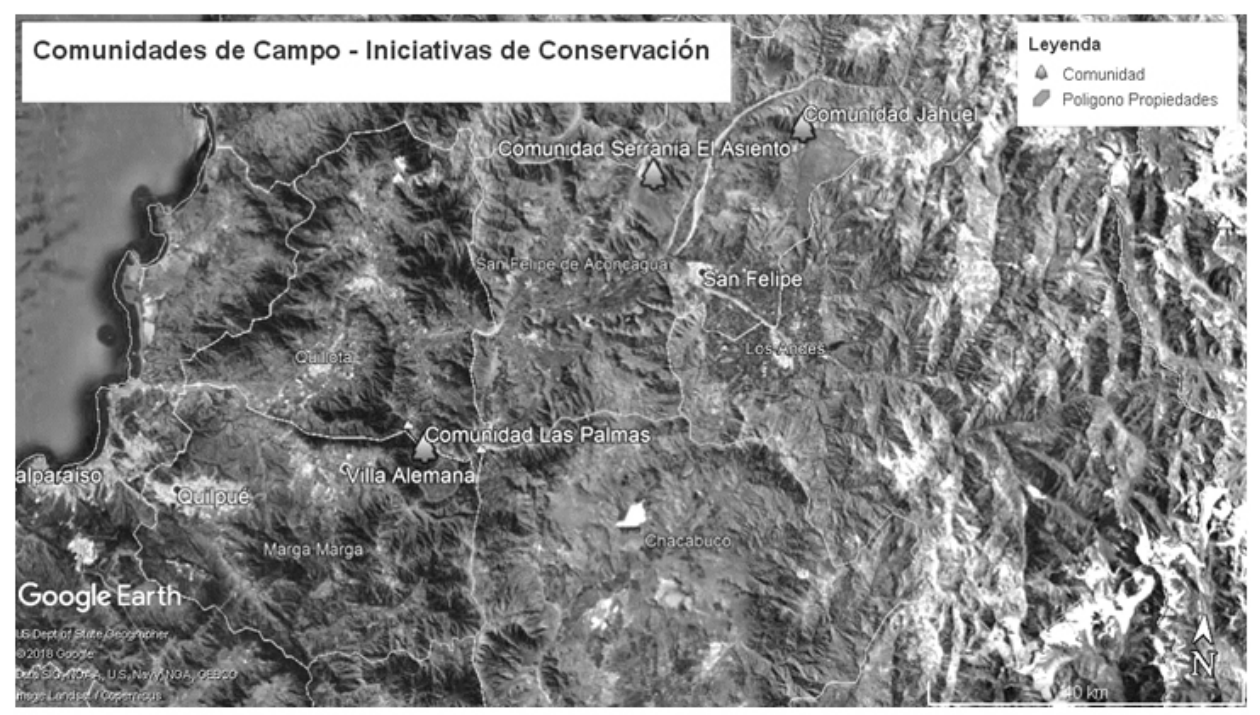

Fuente: Elaboración propia 


\section{Discusión}

Enfrentadas a un contexto de profundas transformaciones derivadas de la expansión del capital, las comunidades experimentan procesos de fragmentación territorial y de resurgimiento de identidades colectivas asociadas, en el caso de los comuneros, a la defensa de los bienes comunes. Se da, en consecuencia, una revalorización de los territorios expresada en "en una tensión continua entre fragmentación y homogeneización" (Nogué, 2007, p. 136). Las comunidades de tierras no vacilan, por ejemplo, levantar sus manos, en asamblea,de manera unánime para rechazar la oferta de compensación que una empresa minera les ofrece como pago por una servidumbre de paso. Con el registro de ese simple gesto, se transita al campo de la política. Lo que resulte de este tipo de decisiones, en la lucha por la hegemonía, dará forma a los paisajes actuales y futuro. Es a través de estos medios, como, al mismo tiempo, las comunidades resisten e intentan ejercer soberanía sobre las tierras comunitarias (Razeto, 2016).

Resulta paradójica desde la perspectiva de la tragedia de los comunes la actitud de las comunidades estudiadas, que activan mecanismos de conservación como una manera de "gravar" sus propias tierras, de restringir y acotar su disfrute en virtud de aspiraciones de mayor alcance. Es el caso de la declaratoria de santuarios de naturaleza que los convierte, no en ecologistas o conservacionistas, sino que, más bien, en gobernantes de su territorio sobre el que buscan ejercitar estrategias de control y de poder soberano.

Las comunidades de tierra han desplegado en plenitud y durante al menos 300 años, prácticas de arriería que constituyenla vivencia del "ser - en las montañas", alineamiento donde la posición propia se define en su relación con la de seres otros que humanos (Descola, 2013). Semejante posicionamiento invita a la comprensión de una "ecología arriera”, como propone Catalán (2015). Se entiende así a los comuneros, portadores de una forma particular de vivenciar la ecología, en tanto habitantes que establecen relaciones recíprocas con el medio en que viven, a la vez que agentes activos en la conservación de sus tierras. Como es evidente, esta visión contesta a la tragedia de los comunes, ya que la ganadería aparece más como un garante de la conservación que, como un agente degradante de los ecosistemas, siendo más gravitantes, en estos casos los factores culturales el sentido de la ganadería-, que los factores biológicos propios de esta actividad. No está de más mencionar que en todas estas comunidades concurren normas más o menos formales respecto a la tenencia ganadera, como parte de su institucionalidad; no obstante, el elemento más notable es que en ninguno de los casos la motivación económica es la principal razón para la tenencia ganadera, lo cual rompe con la razón instrumental moderna argumento que subyace no solo a la tragedia sino que también a otras orientaciones contemporáneas en conservación - dando cuenta de la presencia de lógicas no modernas presentes en estas comunidades.

La acción colectiva desplegada por las comunidades de tierras lo es, como se ha señalado, inspirada por la defensa de sus formas de vida y por los territorios que son parte de su identidad como comuneros y no por intenciones exclusivamente conservacionistas. Las acciones comunitarias, en este sentido, entrañan un conocimiento que se desprende de la vivencia íntima con la montaña y con los ganados, y su ejercicio es consistente con los ciclos que hacen posible la existencia en común de seres humanos, animales, árboles y fuentes de agua. La "ecología arriera” y su defensa por parte de las comunidades involucra pues un elemento de conservación que merece ser entendido a la luz de los muchos engranajes que sostienen esa vida. Tales engranajes se establecen en relación al hábitat, cuya defensa, lo es en consecuencia de sus partes constitutivas y no de una conciencia ecologista o conservacionistas.

Es el apego al ambiente no solo como signo de identidad y de pertenencia a un territorio particular, sino al modo como la relación se ha construido con sus antepasados y su ganado, lo que les ha llevado a desarrollar un profundo sentido de libertad que hace posible la cohabitación cordillerana con los animales (Razeto, 2016). El "ser arriero" supera con creces la visión ecológica convencional; se trata, más allá de las funcionalidades ecológicas, de los modos del habitar en que se ancla, en un período de turbulencia, una búsqueda identitaria, aún incierta, pero que aspira a mantener tales modos, a pesar de las limitaciones que la expansión del capital les impone. Sus ejercicios formales de conservación han de comprenderse, pues, en sintonía identitaria y parecen ser funcionales a sus intenciones, no siendo fines en sí mismos, sino medios estratégicos para continuar siendo quienes son. 
Es evidente que no todos los comuneros comparten los sentidos antes mencionados. Las comunidades son cuerpos sociales heterogéneos, donde las racionalidades se confunden y donde la lógica moderna se encuentra tanto o más presente que aquella que se le opone. Su futuro entonces parece incierto y una mirada prospectiva abre la posibilidad de entender sus formas de vida fundidas y confundidas en medio de la vorágine moderna, con gestos de conservación de la vida arriera, como con elementos de modernización irreversible. Se trata de dinámicas y procesos complementarios, anclados en una realidad fraccionada, diversa, donde todos esos mundos y sus tendencias son posibles. Magia y tragedia, como partes sustantivas de una realidad tan cambiante como inmutable.

Los arrieros no son ecologistas en el sentido de la ecología política de Lipietz (1999), su discurso y sus prácticas se sitúan en las esferas de la disputa por el poder en sus territorios. Lo que se encuentra en pugna en verdad es una contraposición cultural, que hace referencia a la soberanía, al control de las tierras de montaña. Ellos se saben y se sienten protectores del lugar, pero por estar dentro, por ser parte de él (Catalán, 2015; Razeto, 2016). Frente a los argumentos ecológicos formales, ellos desarrollan sus propios argumentos, incluyendo incluso aspectos de dicho discurso ecológico, pero claramente no es la ecología naturalizada o militante de los ecologistas, no es la ecología política de los intelectuales. Es la ecología arriera, siendo ésta tan política como la anterior.

Las iniciativas de conservación que impulsan pueden ser interpretadas como ejercicios básicos de existencia, como expresiónde sus prácticas ganaderas trashumantes que les permiten mantener vivas sus costumbres. Es la libertad, el gusto, el amor, el vínculo, los sentidos del paisaje, lo que les inspira y otorga sentidoa su existencia cotidiana. Es esa misma existencia, la que expresa malestar, indignación y rechazo a las intervenciones externas. Y no son solo lasactividades industriales,sino que la modernidad misma que si bien les atrae, también les ahoga y oprime. Volver a la montaña, mantener las prácticas trashumantes y semi-nómades, mantener una y mil conversaciones en sus fogones, retornar a sus lugares y seguir siendo parte del paisaje, parecen recursos de rebeldía y de libertad (Razeto, 2016; Catalán, 2015). Mantener esas prácticas es una forma entre otras de mantener soberanía sobre sus tierras, es demostrar que están vivos y que son propietarios de ellas.

En su existencia cotidiana los comuneros continúan realizando ganadería trashumante, a pesar que pierden dinero. Continúan con sus paisajes de adoración, cofradías de bailes chinos, encienden velas a la Virgen y hacen rogativas a los santos y santas patronas, para que llueva, para tener agüita para las cosechas, para que salga pasto en las cordilleras, continúan recuperando su historia local, recordando su pasado de valor y resiliencia frente a la adversidad. En un escenario de desigualdades,es suyo el derecho a defender sus tierras, reconociendo en sus relatos, acciones y prácticas, gestos de soberanía sobre sus territorios. Territorio que no se puede dejar de entender cómo simbolizado y significado por las comunidades propietarias que lo ocupan, habitan, recorren y vivencian de manera particular. Giménez (2007) ilumina la imagen de identidades socialmente territorializadas, "para encuadrar adecuadamente los fenómenos de arraigo, apego y sentimientos de pertenencia socioterritorial”(p. 151). Fenómenos que permiten dar cabida a la comprensión de las acciones de apropiación y defensa de los territorios montañeses que realizan las comunidades.

El capitalismo y su lógica de mercantilización de la naturaleza son lo que se yergue sobre las montañas y comunidades de la región(Leff, 2004). Son nuevas las formas de desposesiónque se imponen a los comuneros. En efecto, la tierra, el subsuelo y el agua son objetos de la voracidad del capital y, en tanto, las comunidades son propietarias de territorios donde se les encuentra, por lo que la acumulación por desposesión es condición necesaria para la expansión mercantil (Harvey,2011; Razeto, 2016). La institucionalización de la desposesión como mecanismo del sistema dominante cobra plena vigencia en este territorio: sea por la des-regulación en el acceso a los recursos mineros, sea por las cesiones de derechos públicos como el agua, sea por la liberalización de los controles ambientales, se erosiona la propiedad en común (Guerra y Skewes, 2010). Es, a la inversa de lo sospechado, la propiedad privada individual la que procura hacerse del agua y de los recursos del subsuelo y, como es habitual, de exportar sus externalidades. La acción de los arrieros merece ser tenida como contraposesiva,resistentey tremendamente efectiva hasta ahora. Se cumple con la condición según la que "bajo discursos y expresiones que resultan de la simbiosis entre comunidad y paisaje, que se traducen en formaciones o regímenes ecológicos que se tornan relativamente inexpugnables o, al menos circunstancialmente soberanos” 
(Ibid, p. 33). Estas formas de resistencia, no obstante, resultan frágiles y, de no prosperar, generarían transformaciones radicales en la ecología local.

La experiencia colectiva de los comuneros de la región mediterránea de Chile sugiere que la recreación de la vegetación nativa no supone la exclusión de los seres humanos, sino que, por el contrario, la presencia de los seres humanos puede ser una condición favorable para que tal regeneración se produzca. Los comuneros permiten avanzar en la respuesta acerca de cuáles son las avenidas que la hacen posible. Tres son, por lo menos, las rutas que se desprenden de la discusión precedente. La primera de ellas tiene que ver con la gestión colectiva de las tierras en común. Siendo Chile uno de los países con menor porcentaje de tierras comunitarias, o tierras designadas para la administración comunitaria, a nivel mundial -tan solo un 3,12\% de la superficie nacional, mientras que la tendencia en América Latina es del 23,19\% (Rights and Resources Initiative, 2015)- resulta sintomático constatar que tales tierras se encuentran más protegidas de lo que lo están las caen bajo la propiedad privada. Esta situación se deriva de la naturaleza colectiva del quehacer comunero. No se postula aquí una mutación ontológica que haga a los arrieros partícipes de una realidad sustantivamente diferente del campesinado en general, sino que, más bien, de un vínculo sustantivo con la montaña, los animales, las aguas y la vegetación. No son, en la perspectiva de los comuneros, recursos. Son más bien compañeros de ruta en la reproducción de la vida. Si esto es o no una otra ontología no es algo que pueda resolverse aquí, pero lo que sí queda de manifiesto es el vínculo identitario y afectivo que sostiene el arreglo colectivo más allá de los soportes institucionales que Ostrom sostiene como necesarios.

A pesar del protagonismo que los comuneros han desplegado en la defensa de sus territorios, avalados por el vínculo afectivo al que se hacía alusión, no es menos cierta la amenaza que se cierne sobre ellos a partir de la expansión del capital sea por la vía de la agricultura intensiva, la minería o la expansión inmobiliaria, fenómenos que sobrepasan las posibilidades de los habitantes locales. La comprensión del papel ecológico que asume el comunero, tal como se desprende de este artículo, impone una lectura desde el Estado que lo identifique no como un objeto de política sociales sino como un garante de la supervivencia del bosque Chile.

\section{Bibliografía}

Agrawal, A. (2007). Forests, governance, and sustainability: common property theory and its contributions. International journal of the commons, 1(1), 111-136. doi: http://doi.org/10.18352/ijc.10

Alimonda, H. (2016). Notas sobre la ecología política latinoamericana: arraigo, herencias, diálogos. Ecología Política, (51), 36-42. Recuperado de https://www.ecologiapolitica.info/?p=6017

Alimonda, H., Toro Pérez, C. y Martín, F. (Eds.). (2017). Ecología política latinoamericana. Buenos Aires, Argentina: UAM/CLACSO.

Baraona, R., Aranda, X., y Santana, R. (1961). Valle de Putaendo. Estudio de estructura agraria. Santiago, Chile: Ed. Universidad de Chile.

Bautista Segales, J. J. (2014). ¿Qué significa pensar desde América Latina? Madrid, España: Akal.

Bengoa, J. (1990). El Poder y la Subordinación: Historia Social de la Agricultura Chilena.Santiago, Chile: Ediciones SUR.

Booth, K. I. (2013). Deep Ecology, Hybrid Geographies, and Environmental Management’s Relational Premise. Environmental Values, 22(4), 523-543. doi: https://doi.org/10.3197/096327113X13690717320829

Borde, J., y Góngora, M. (1956). Evolución de la propiedad rural en el valle del Puangue (Vol. 1). Santiago, Chile: Editorial Universitaria. 
Brucher, M.E. (1962) Estudio socio-económico de la comunidad agrícola de Caleu(Tesis de pregrado). Universidad de Chile, Santiago, Chile.

Carvajal, D. (2014). Conflicto de contenido ambiental, acción colectiva y transformaciones en la cultura política local: El caso de la defensa del Valle de Jahuel(Tesis de pregrado). Universidad de Chile, Santiago, Chile.

Castro, M., y Bahamondes, M. (1986). Surgimiento y transformación del sistema comunitario: Las comunidades agrícolas, IV Región, Chile. Ambiente y Desarrollo, 2(1), 111-126. Recuperado de https://www.opia.cl > articles-75229_archivo_01

Catalán, E. (2015). Relaciones humano-ambiente en el Parque Nacional La Campana: Una trayectoria de encuentros y desencuentros entre Comunidades Locales y el Área Protegida(Tesis de pregrado). Universidad de Chile, Santiago, Chile.

Ciem Aconcagua (2015a). Plan de Manejo Complejo El Zaino - Laguna El Copín. San Felipe, Chile: Corporación Ciem Aconcagua.

(2015b) Plan de Manejo Complejo Santuario de la Naturaleza Serranía del Ciprés. San Felipe, Chile: CorporaciónCiem Aconcagua.

Costanza, R. (2000).Social Goals and the Valuation of Ecosystem Services. Ecosystems, 3(1), 4-10. doi: https:// doi.org/10.1007/s100210000002

Cowling, R. M., Rundel, P. W., Lamont, B. B., Arroyo, M. K. y Arianoutsou, M. (1996). Plant diversity in Mediterranean-climate regions. Trends in Ecology \& Evolution, 11, 362-368. doi:

Cox, R. L. y Underwood, E. C. (2011). The importance of conserving biodiversity outside of Protected Areas in Mediterranean ecosystems: Plos One, 6, E14508. doi: Descola, P. (2013). Beyond nature and culture. [Traducido al inglésPar-delà nature et culture]. Chicago: University of Chicago Press.

Elórtegui, S. y Moreira-Muñoz, A. (eds.)(2009). Parque Nacional La Campana. Origen de una Reserva de la Biosfera para Chile Central. Viña del Mar, Chile: Taller La Era.

Erazo, M. B. y Garay-Flühmann, R. (2011). Tierras secas e identidad. Una aproximación cultural a las prácticas de subsistencia de las comunidades campesinas del semiárido: Provincia de Elqui, Chile. Revista de Geografía Norte Grande, (50), 45-61. doi: http://dx.doi.org/10.4067/S0718-34022011000300004.

Escobar, A.(2010). Territorios de diferencia: lugar, movimientos, vida, redes. Popayán, Colombia: Envión Editores.

(2016). Autonomía y diseño. La realización de lo comunal. Popayán, Colombia: Editorial Universidad del Cauca.

Gajardo, A. (2014). 4. Etnicidad, reemergencia indígena y conflicto minero. El proyecto Pascua Lama, el Estado y el proceso de reetnificación de los Diaguitas del Huasco Alto, Chile. Anuario del conflicto Social, (4). Recuperado de http://revistes.ub.edu/index.php/ACS/article/view/12276

Gatica, T y Thayer, C. (1970). Reforma Agraria y Pequeña Propiedad: estudio del área Aconcagua y Putaendo. Investigaciones Geográficas $N^{\circ} 20$. Santiago, Chile: Ed. Universidad de Chile.

Giménez, G. (2007). Estudios Sobre la Cultura y las Identidades Sociales. México: ITESO. doi: 10.2307/j.ctvdmwzr4

Guerra, D. y Skewes, J.C. (2010). Acumulación por desposesión y respuestas locales en el remodelaje de los paisajes estuariales del Sur de Chile. Chungará, 42: 451- 463. doi: http://dx.doi.org/10.4067/S0717- 


\section{8}

Guerra, J.P. (2005). Pastoreo Trashumante en el Valle del Aconcagua(Tesis de pregrado).Universidad Academia de Humanismo Cristiano, Santiago, Chile.

Hardin, G. (1968). The Tragedy of the Commons. Science, 162(3859), 1243-1248. doi:

Harvey, D. (2011). El ‘nuevo’ imperialismo: acumulación por desposesión.Socialist Register 2004, 99-129. Recuperado de https://socialistregister.com/index.php/srv/article/view/14997/11983

Hess, C., y Ostrom, E. (eds.) (2011). Understanding knowledge as a common: from theory to practice. Cambridge, UK: MIT Press.

Heywood, P. et al. (2017). The Ontological Turn. Cambridge Encyclopedia of Anthropology.doi: https://doi.org/ 10.29164/17ontology

Hidalgo, R., Borsdorf, A., y Plaza, F. (2009). Parcelas de agrado alrededor de Santiago y Valparaíso: ¿Migración por amenidad a la chilena? Revista de Geografía Norte Grande, (44), 93-112. doi: http://dx.doi.org/10.4067/ S0718-34022009000300005

Leff, E. (2004). Racionalidad Ambiental: la reapropiación social de la naturaleza.Ciudad de México, México: Siglo XXI.

(2017). Las relaciones de poder del conocimiento en el campo de la ecología política: una mirada desde el sur. En Alimonda, H., Toro Pérez, C. y Martín, F. (eds.). (2017). Ecología política latinoamericana (pp.129165). Buenos Aires, Argentina: UAM/CLACSO.

Lipietz, A. (1999). Qu'est-ce que l'écologie politique? La grande transformation du XXI siècle. París, Francia: La decouverte.

Livenais, P. y Aranda, X. (2003). Dinámicas de los sistemas agrarios en Chile Árido: La Región de Coquimbo. Santiago, Chile: Lom Editores.

Ministerio de Bienes Nacionales (s.f.) Regularización de Comunidades Agrícolas. Recuperado de http:// www.bienesnacionales.cl/?page_id=1887

Nogué, V. (2007). Paisaje, identidad y globalización. Fabrikart, (7) 136-144.

Ostrom, E. (2009). A general framework for analyzing sustainability of social - ecological systems. Science, 325, 419-422. doi: 10.1126/science.1172133

Parra-Romero, A. (2016). ¿Por qué pensar un giro decolonial en el análisis de los conflictos socioambientales en América Latina? Ecología política, (51), 15-20. Recuperado de https://www.ecologiapolitica.info/?p=6006

Patzi, P. (2004). Sistema comunal: una propuesta alternativa al sistema liberal: una discusiónteórica para salir de la colonialidad y del liberalismo. La Paz, Bolivia: Comunidad de Estudios Alternativos CEA.

Razeto J. (1986). Economía Campesina Comunitaria: dos ejemplos para un análisis(Tesis de pregrado). Universidad de Chile, Santiago, Chile.

2016. Comunidades, lugares, paisajes y tensiones en las montañas de Aconcagua en Chile Central(Tesis doctoral). Universidad Nacional de Cuyo, Mendoza, Argentina.

Rights and Resources Initiative. (2015). Who Owns the World's Land? A global baseline of formally recognized 
indigenous and community land rights.Washington, DC: RRI. Recuperado de https://rightsandresources.org/ es/who-owns-the-worlds-land-a-global-baseline-of-indigenous-and-community-land-rights/\#.XSq7B3t7nIU

Rivera Cusicanqui, S. (Junio de 2017). Comunalidades anarquistas. Una aproximación testimonial. En E. Restrepo (Presidencia). Éticas y políticas alternativas a la academia. Debate central realizado en el V Congreso de Asociación Latinoamericana de Antropología, Bogotá, Colombia. Recuperado de http:// periodicoellibertario.blogspot.com/2017/07/bolivia-comunalidades-anarquistas-una.html

Schulz, J. J., Cayuela, L., Echeverría, C., Salas, J. y Rey Banayas, J. M. (2010). Monitoring land cover change of dryland forest landscape of Central Chile (1975-2008). Applied Geography, 30, 436-447. doi:

Simonetti, J. A. (1999). Diversity and conservation of terrestrial vertebrates in mediterranean Chile. Revista Chilena de Historia Natural, 72, 493-500. Recuperado de http://rchn.biologiachile.cl/pdfs/1999/4/ Simonetti_1999.pdf

Solís de Ovando, J. (2004). Normativa Legal de las Comunidades Agrícolas: análisis crítico del DFL n 5 de 1968 del Ministerio de Agricultura. Santiago, Chile: Grupo de Investigaciones Agrarias GIA.

Stake, R. E. (2006) Multiple Case Study Analysis. Nueva York, Estados Unidos: Guilford Press.

Tognelli, M. F., Ramírez de Arellano, P. I. y Marquet, P. A. (2008).How well do the existing and proposed reserve networks represent vertebrate species in Chile? Diversity and Distributions, 14, 148-158. doi: https://doi.org/ 10.1111/j.1472-4642.2007.00437.x

Venegas, F. (2009). Los Herederos de Mariana Osorio. Comunidades Mestizas de Olmué: Repúblicas campesinas en los confines del Aconcagua Inferior, ss. XVII - XXI. Olmué, Chile: Comunidad Agrícola-Ganadera Mariana de Osorio Olmué Granizo.

Vergara, R. (2005). Población y Asentamientos Humanos en el Ámbito de las Comunidades Agrícolas - Región de Coquimbo.Santiago, Chile: Instituto Nacional de Estadísticas INE. 